\title{
Poster Abstract: Long Term Occupancy Estimation in a Commercial Space: An Empirical Study
}

\author{
Kin Sum Liu \\ Stony Brook University \\ kiliu@cs.stonybrook.edu
}

\author{
Sirajum Munir \\ Bosch Research and Technology \\ Center, Pittsburgh, PA \\ sirajum.munir@us.bosch.com
}

\author{
Jonathan Francis \\ Bosch Research and Technology \\ Center, Pittsburgh, PA \\ jon.francis@us.bosch.com
}

\author{
Charles Shelton \\ Bosch Research and Technology \\ Center, Pittsburgh, PA \\ charles.shelton@us.bosch.com
}

\author{
Shan Lin \\ Stony Brook University \\ shan.x.lin@stonybrook.edu
}

\begin{abstract}
Understanding occupancy patterns in a building is very useful to control HVAC systems for improving energy efficiency of the building and occupant comfort. There has been a very little attempt to understand long term occupancy patterns in a commercial space. In this work, we leverage depth sensors (Kinect for XBOX One) to collect occupancy count from an 11,000 square foot commercial space (Bosch office) for nine months. We analyze the collected data and describe key findings that provide deep insights about how a commercial space is used and serve as a guideline to formulate novel and efficient control strategies.
\end{abstract}

\section{CCS CONCEPTS}

-Computer systems organization $\rightarrow$ Sensor networks; $\bullet$ Hardware $\rightarrow$ Temperature control; Impact on the environment;

\section{KEYWORDS}

Occupancy Prediction, HVAC Control, Occupancy Data Collection

\section{ACM Reference format:}

Kin Sum Liu, Sirajum Munir, Jonathan Francis, Charles Shelton, and Shan Lin. 2017. Poster Abstract: Long Term Occupancy Estimation in a Commercial Space: An Empirical Study. In Proceedings of The 16th ACM/IEEE International Conference on Information Processing in Sensor Networks, Pittsburgh, PA USA, April 2017 (IPSN 2017), 2 pages.

DOI: http://dx.doi.org/10.1145/3055031.3055062

\section{INTRODUCTION}

Heating, ventilation, and air conditioning (HVAC) is a major source of energy consumption in the US. In 2006, approximately $35 \%$ of energy in the US was used for HVAC [1]. Usually building operators use a static schedule for controlling HVAC systems without having a deeper understanding of how many people use the building at different times of the day. In addition, some HVAC systems operate by assuming maximum occupancy in each room, which leads to

Permission to make digital or hard copies of all or part of this work for personal or classroom use is granted without fee provided that copies are not made or distributed for profit or commercial advantage and that copies bear this notice and the full citation on the first page. Copyrights for components of this work owned by others than ACM must be honored. Abstracting with credit is permitted. To copy otherwise, or republish, to post on servers or to redistribute to lists, requires prior specific permission and/or a fee. Request permissions from permissions@acm.org.

IPSN 2017, Pittsburgh, PA USA

(c) 2017 ACM. 978-1-4503-4890-4/17/04 ..\$15.00

DOI: http://dx.doi.org/10.1145/3055031.3055062 a significant energy waste, e.g., an HVAC system providing ventilation for 30 people when there are only 10 people in a room [2]. Understanding how many people use different rooms at different times of the day is very crucial for improving energy efficiency and occupant comfort.

There have been a few attempts [4] to collect long term occupancy patterns from office buildings. However, PIR motion detectors are used in such projects for sensing occupancy and hence the datasets only reflect whether a room is occupied or not without revealing the actual person count. Only a few datasets [2] capture actual person count from commercial spaces, and even in these cases, the data collection period was very limited. We are the first to collect long term (9 months) occupancy data (people count) from an 11,000 square foot commercial office (second floor of Bosch Research and Technology Center Pittsburgh). In this paper, we outline some statistical results from our empirical study and explore their implications for saving energy.

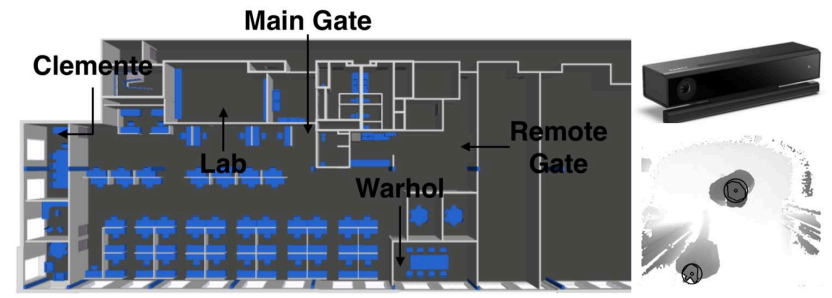

Figure 1: Floor plan of the office and the depth sensor used in FORK with a sample depth image

\section{DEPLOYMENT, DATA COLLECTION}

We use a depth sensor (Kinect for XBox One) for counting number of people in a room. The depth sensor is mounted on the ceiling looking downwards near to a doorway as it accurately counts the number of people entering and exiting through the door. The solution is called FORK (Fine grained Occupancy estimatoR using Kinect) and the detailed algorithm is described in [3]. A FORK depth sensor and a sample depth image are shown at the right side of Figure 1. We have deployed five instances of FORK at a Bosch office (the floorplan is shown at the left side of Figure 1) to cover the common office space (requires monitoring at two entrances: Main Gate and Remote Gate), two conference rooms (Warhol and Clemente), and a lab. Warhol and Clemente conference rooms are the most used and largest conference rooms of this office that can 

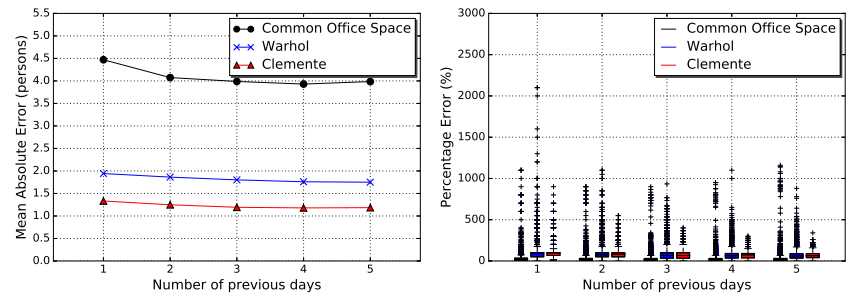

(a) Using previous days for prediction
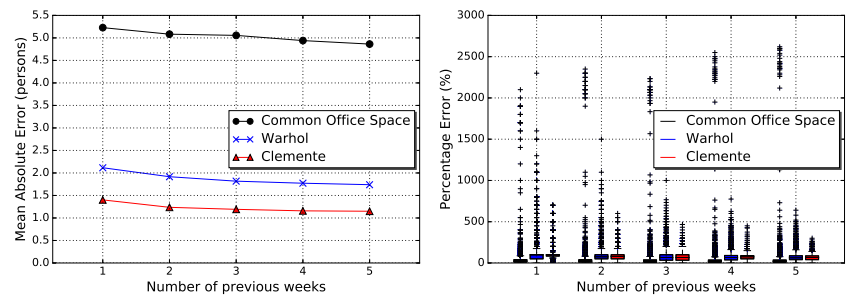

(b) Using previous weeks for prediction

Figure 2: Prediction errors when using historical data to predict occupancy count from 9:00 to 18:00

accommodate 25 and 15 people, respectively. After collecting over 9 months of data, we stopped the data collection on June 7th 2016. During this period, there were 64925, 18477, and 9938 entrance and exit events in the common office space (Main Gate and Remote Gate), Warhol, and Clemente conference rooms, respectively.

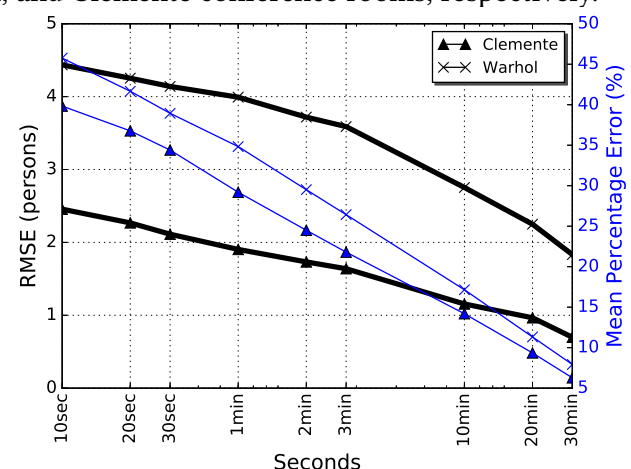

Figure 3: Prediction errors on number of participants in meetings based on initial people count

\section{FINDINGS}

The findings from the empirical study are about understanding and predicting occupancy patterns $(1,2,3,4)$, understanding the usage of the office space $(5,6)$ and conference rooms $(7,8)$, and weekend behavior (9).

(1) The daily occupancy pattern of the common office space is regular. However, the percentage change of the maximum occupancy compared to the previous day varies from $0 \%$ to $730 \%$ with an average of $18.4 \%$, which translates to 4.1 people on average.

(2) The daily occupancy patterns in conference rooms are very irregular.

(3) Simple solution leveraging occupancy count from the previous day for predicting future number of occupants suffers a percentage error from $0 \%$ to $1100 \%$ with an average of $30.1 \%$ for the common office space, which translates to an RMSE of 5.95 people on average (Fig. 2(a)). It is much worse for the conference rooms, e.g., in Warhol it varies from $0 \%$ to $2100 \%$ with an average of $104 \%$. Leveraging more than two days of historical data does not improve occupancy prediction significantly. Using the same weekdays of the previous weeks suffers from a larger error in the common office space (Fig. 2(b)).

(4) Number of people at the initial 3 minutes of a meeting is a useful indicator for predicting number of participants of that meeting (Fig. 3). Such a simple predictor achieves 2.6 people RMSE on average.
(5) Arrival time of the earliest person is 7:25 AM on average, which is 1.5 hours before the arrival of the earliest group of people (10 people in this analysis).

(6) Departure time of the latest person is 8:43 PM on average, which is 3 hours later than the latest group.

(7) In conference rooms, the average and median number of participants in meetings are 3.85 and 3, respectively. We find that in $99 \%$ cases, the number of participants does not reach the maximum occupancy capacity. In fact, it does not exceed half of the capacity in $96 \%$ cases. The average and median durations of meetings are 63 and 48 minutes, respectively.

(8) The utilization of conference rooms varies at different times of the day with an average of $55.6 \%$ between 9 AM and 6 PM, which means conference rooms are unused for $44.4 \%$ of the time on average.

(9) The office space is not completely unused during weekends. The probability of occupancy in the common office space is $57.9 \%$ and the utilization of conference rooms is $4.8 \%$ on average during a weekend day.

\section{CONCLUSIONS}

Occupancy count of the common office space shows a periodic pattern with varied amplitudes (maximum occupancy) due to dynamic behavior of the occupants, e.g., joining or departure of interns, arrival of visitors, and hosting of workshops. Hence, an adaptive prediction scheme may be useful. However, occupancy prediction is very difficult for conference rooms. Since occupancy in the first 3 minutes is a useful indicator of the number of meeting participants, a multistage HVAC system can be useful. We see that the variance of the earliest arrival and latest departure time for an individual is much larger than that of a group behavior. Hence, if we sacrifice the comfort of the first and last few people, that can reduce energy consumption significantly.

\section{ACKNOWLEDGMENTS}

This work was supported, in part, by DOE grant DE-EE0007682. The opinions expressed here are from the authors and do not necessarily reflect the views of DOE.

\section{REFERENCES}

[1] EIA. http://www.eia.doe.gov/.

[2] Varick L. Erickson, Miguel Á. Carreira-Perpiñán, and Alberto Cerpa. 2011. OBSERVE: Occupancy-based system for efficient reduction of HVAC energy. In IPSN.

[3] S. Munir, R. S. Arora, C. Hesling, J. Li, J. Francis, C. Shelton, C. Martin, A. Rowe, and M. Berges. 2017. Real-Time Fine Grained Occupancy Estimation using Depth Sensors on ARM Embedded Platforms. In RTAS.

[4] C.R. Wren, Y.A. Ivanov, D. Leigh, and J. Westhues. 2007. The MERL Motion Detector Dataset. In Workshop on Massive Datasets (MD). 